\title{
Role of Plasma Elongation on Turbulent Transport in Magnetically Confined Plasmas
}

\author{
P. Angelino, ${ }^{1}$ X. Garbet, ${ }^{1}$ L. Villard, ${ }^{2}$ A. Bottino, ${ }^{3}$ S. Jolliet, ${ }^{2}$ Ph. Ghendrih, ${ }^{1}$ V. Grandgirard, ${ }^{1}$ B. F. McMillan, ${ }^{2}$ Y. Sarazin, ${ }^{1}$ \\ G. Dif-Pradalier, ${ }^{1}$ and T.M. Tran ${ }^{2}$ \\ ${ }^{1}$ Association Euratom-CEA, CEA/DSM/IRFM, Cadarache, France \\ ${ }^{2}$ CRPP Lausanne, Association Euratom-Confédération Suisse, Lausanne, Switzerland \\ ${ }^{3}$ Max Plank Institut für Plasmaphysik, IPP-EURATOM Association, Garching, Germany
}

(Received 26 September 2008; published 12 May 2009)

\begin{abstract}
The theoretical study of plasma turbulence is of central importance to fusion research. Experimental evidence indicates that the confinement time results mainly from the turbulent transport of energy, the magnitude of which depends on the turbulent state resulting from nonlinear saturation mechanisms, in particular, the self-generation of coherent macroscopic structures and large scale flows. Plasma geometry has a strong impact on the structure and magnitude of these flows and also modifies the mode linear growth rates. Nonlinear global gyrokinetic simulations in realistic tokamak magnetohydrodynamic equilibria show how plasma shape can control the turbulent transport. Results are best described in terms of an effective temperature gradient. With increasing plasma elongation, the nonlinear critical effective gradient is not modified while the stiffness of transport is decreasing.
\end{abstract}

PACS numbers: 52.55.Fa, 52.30.- q, 52.35.Ra, 52.65.-y

One of the main difficulties plasma physics research must face in order to obtain self-sustained fusion reactions is caused by the energy losses due to turbulent transport, resulting in a reduction of the energy confinement time. Therefore great effort is put toward investigating the physics mechanisms underlying turbulent transport in order to find a way to control and reduce it. Despite this effort, many questions remain unanswered, mainly because of the complexity and nonlinearity of the phenomenon. Experimentally a set of scaling laws has been identified which relate the energy confinement time to several global plasma parameters, but a theoretical model, capable of fully explaining these laws, is still missing. In these scaling laws, plasma geometry is found to play a crucial role in the determination of the turbulent transport level. In particular, the elongation (defined as the ratio between the vertical and the horizontal size of the plasma cross section) has an exponent close to unity [1]. In the framework of gyrokinetic theory, the problem has been tackled by linear stability analysis [2-4] and nonlinear local flux tube simulations $[5,6]$.

In this Letter, a new analysis scheme is proposed where linear and nonlinear effects can be separated, in an effort to bring more insight into the physical mechanisms relating transport to geometry. While nonlinear terms can easily be dropped in the equations leading to a linear stability analysis, the difficulty resides in subtracting the linear effects from nonlinear simulations. The idea is to choose a combination of physical and geometrical parameters such that, when expressed in these terms, the effects of elongation vanish in linear simulations. If effects of elongations are still observed in nonlinear simulations expressed in the same parameter combination, they are of nonlinear nature.

A second original feature of the analysis presented is that, for the first time, the problem is tackled by means of a full global code. Therefore, the consistent effects of the elongation profile variation are taken into account, not only its local value or its local shear value. Previous works $[5,6]$ have made use of a local parametrized equilibrium model where the elongation and its shear appear as independent parameters. Another open question is whether core or edge turbulence is the relevant factor in the observed scaling of transport with elongation. Because of model limits, the study presented here is restricted to the plasma core. For the case of edge turbulence we refer to the work by Kendl and Scott [7], where very strong dependence of the edge turbulence driven transport on elongation has been reported.

The ion thermal diffusivity is found to scale inversely with the plasma elongation, in qualitative agreement with previous findings using local flux tube simulations. The linear results can be understood by a modification of the driving temperature gradient induced by the plasma shape, leading to a linear up-shift of the critical temperature gradient. Nonlinearly, geometry comes into play by modifying the self-generated flows which contribute to the turbulence saturation. In Ref. [8] the strong impact of elongation on large scale structures has been shown, zonal flows (ZFs) in particular, which suggests a stabilizing effect on turbulence.

This Letter aims to show how geometry and the large scale structures of self-organized turbulence could be used to control the transport property of a turbulent media. This question is addressed here with global gyrokinetic simulations run with the code ORB5 [9]. The magnetic equilibria, giving the geometry of the problem, are supplied by the toroidal axisymmetric equilibrium solver code CHEASE [10]. The geometry is therefore that of true ideal MHD equilibria as provided by a numerical solution of the GradShafranov equation. Noting $r$ as a label for the magnetic 
surfaces, the elongation is given by a function $\kappa(r)$. Four values of the edge elongation are considered $\kappa_{b}=1,1.25$, $1.5,1.75$, ranging from a circular plasma to the elongation chosen for ITER. Other relevant physical parameters for this set of simulations are the minor radius on equatorial plane $a=0.48 \mathrm{~m}$, the inverse aspect ratio of $a / R=0.36$, the vacuum magnetic field at the center of the discharge $B_{0}=1.91 \mathrm{~T}$, and the normalized Larmor radius $\rho_{*}=$ $\rho_{s} / a=1 / 140$, where $\rho_{s}=c_{s} / \Omega_{i}, \quad c_{s}=\sqrt{T_{e} / m_{i}}$, and $\Omega_{i}=Z_{i} e B / m_{i}$. The safety factor profile is a second-order polynomial with values $q_{s}(0)=0.85$ at the center and $q_{s}(a)=3.0$ at the edge. Ions are deuterium. The initial temperature profile has a gradient which is constant in the interval $0.2<r<0.8$ and fades to zero outside. Note that ORB5 is a truly global code in the sense that not only the gyrokinetic equations are solved in the whole plasma from the magnetic axis to the edge, but also all profiles are consistent and their time evolution is unconstrained: profile relaxation effects are therefore fully included.

The first question we address is the effect of elongation on the linear drive of the turbulence. The linear drive of the microinstabilities comes from the spatial gradients of density and temperature. Since the linear model decouples the toroidal mode numbers, for each simulation a single toroidal mode number $n$ is explored. A scan in $n$ is needed to provide the full toroidal spectrum of the ion temperature gradient (ITG) modes. The results of this scan are plotted in Fig. 1 , for $R / L_{T}=7.5$, where $R / L_{T}=-(R / T) d T / d r$ is the temperature gradient characteristic length on the midplane. The linear growth rates are reduced with increasing elongation, the spectrum is broadened, and the maximum growth rate is shifted toward higher $n$ values.

A scan in both $\kappa$ and $R / L_{T}$ has been performed. The linearly most unstable mode has been chosen and its behavior as a function of $\kappa$ and $R / L_{T}$ is shown in Fig. 1. The stabilizing effect of elongation is evidenced by a reduction of $\gamma$ for all values of the temperature gradient, and by a

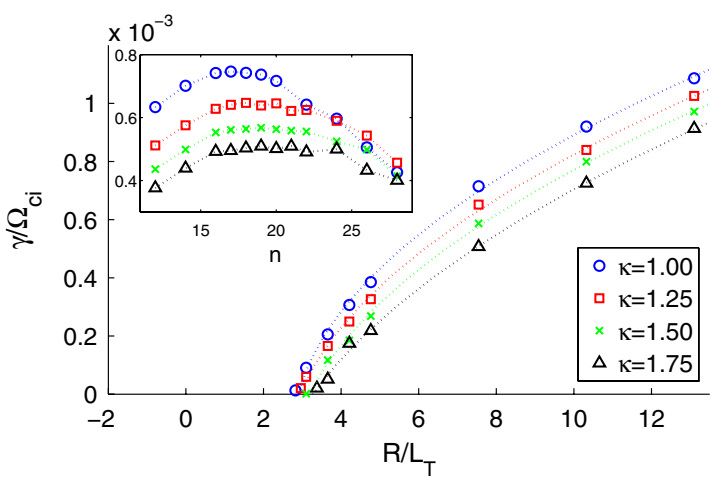

FIG. 1 (color online). Linear growth rates of the linearly most unstable global ITG mode as a function of the temperature gradient scale length $R / L_{T}$ and of the edge elongation. The stabilizing effect of the elongation is observable for all values of the temperature gradient. ITG linear growth rates as a function of the toroidal mode number $n$ are plotted in the top-left corner. Growth rates are normalized to the ion cyclotron frequency $\Omega_{\text {ci }}$. linear up-shift of the critical gradient. Arguments based on the local dispersion relation fail to explain the results from the linear global gyrokinetic simulations, since they would yield a destabilizing effect of the elongation, thus suggesting that the effect we are investigating is of a global nature. We proceed therefore analyzing the global effect of the elongation on the temperature profile.

When considering shaped plasmas, the question arises about which radial coordinate should be used in the definition of the temperature scale length $R / L_{T}=$ $-(R / T) d T / d r$. Since the temperature is taken constant on magnetic surfaces, the shape of the magnetic surface induces a stretching in the temperature gradient. The global ITG mode consequently "feels" an average gradient which is less than the one defined locally on the low field side (LFS) and which appears in the local dispersion relation. It will be shown that this averaged gradient is the relevant one for the global description of turbulence. Formally, the logarithmic gradient can be written as a function of any magnetic surface label coordinate $r$. With an elliptic cross section and the analytic equilibrium described in [11], the coordinate $r_{\text {vol }} \equiv \sqrt{V(\psi) /\left(2 \pi^{2} R\right)}$, where $V(\psi)$ is the volume inside the magnetic surface $\psi=$ const, and the geometric radius on the low field side $r_{\mathrm{LFS}}$ are related by $d r_{\mathrm{LFS}}=-\left(1-E^{\prime}\right) d r_{\mathrm{vol}}$, where $E=r(\kappa-1) /(\kappa+1)$ is a function of the elongation radial profile. Thus

$$
R \frac{d \ln T_{i 0}}{d r_{\mathrm{vol}}}=\frac{R}{T_{i 0}} \frac{d T_{i 0}}{d r_{\mathrm{LFS}}} \frac{d r_{\mathrm{LFS}}}{d r_{\mathrm{vol}}}=-\frac{R}{L_{T i}}\left(1-E^{\prime}\right),
$$

where $\left(R / T_{i 0}\right)\left(d T_{i 0} / d r_{\mathrm{LFS}}\right)=-\left(R / L_{T i}\right)$ is the usual temperature scale length appearing in the local dispersion relation, which now has to be substituted by the expression in Eq. (1). It can be shown that Eq. (1) is indeed equivalent to taking the geometric average of the temperature gradient. According to Eq. (1), the results in Fig. 1 are better understood if plotted as a function of an effective temperature scale length $R / L_{T}\left(1-E^{\prime}\right)$. In terms of this effective gradient, the results corresponding to the four values of $\kappa_{\text {edge }}$ fall on the same curve (Fig. 2), showing the correctness of the above assumption. Additional support of this interpretation comes from the observation that the mode amplitude peaks on the low field side, as is typical for ITG modes, but it extends poloidally at least from $-\pi / 2$ to $+\pi / 2$. It is therefore not surprising that the global mode feels an averaged gradient. Incidentally, it is remarkable that the factor $\left(1-E^{\prime}\right)$ can be well approximated by the factor $1 / \sqrt{\kappa}$, which is obtained by taking the geometric average of the minor radius. We note that the linear results for the cases without triangularity presented in Fig. 6 of Ref. [6] can also be explained in terms of the rescaled gradient, $R /\left(L_{T} \sqrt{\kappa}\right)$.

Given the effect of plasma elongation on the linear stability of ITG modes, the question which arises next is which role is played by this parameter in a nonlinear saturated turbulent regime. In particular, the aim is to find whether there are effects beyond the reduction of the linear 


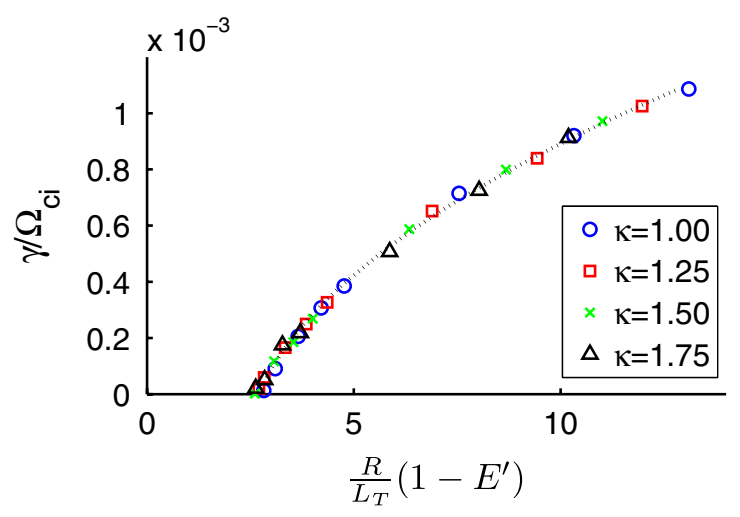

FIG. 2 (color online). Linear growth rates of the linearly most unstable global ITG mode as a function of the effective temperature gradient scale length $R / L_{T}\left(1-E^{\prime}\right)$. Growth rates are normalized to the ion cyclotron frequency $\Omega_{\mathrm{ci}}$.

growth rates analyzed above. To separate clearly the linear and nonlinear effects in a saturated regime could be an arduous task, since by definition the saturated regime is the result of the competition of linear driving terms which feed the turbulence and nonlinear saturation mechanisms which act to quench it. In the present study of the effect of elongation, a solution comes from the analysis of the linear results. According to these, a region can be chosen in the two parameter space of elongation and gradient length so that, from the viewpoint of linear growth rates, the effect of the elongation can be exactly compensated by an increase in the temperature gradient. The result is a set of simulations with the same linear drive, and where differences in the nonlinear saturation mechanisms, if any, could be analyzed.

In practice, an operative value of $\gamma$ and of the corresponding effective temperature scale length $R / L_{T}\left(1-E^{\prime}\right)$ can be chosen with the help of the results of Fig. 2. Any combination of $R / L_{T}$ and elongation which gives the requested value of the effective temperature scale lengths satisfies our requirement of fixed linear drive. For the simulations presented in the following, the value chosen is $R / L_{T}\left(1-E^{\prime}\right)=7.5$. Two elongations are considered for these nonlinear simulations, $\kappa_{b}=1$ and 1.75 , and the normalized Larmor radius is $\rho_{*}=1 / 256$. To fully understand the results plotted in Fig. 3, we must recall that no heat source is present in the model used for these simulations. The heat flux therefore induces a relaxation of the temperature gradient, which approaches the critical value causing a decaying of the turbulence. As ORB5 is a particlein-cell code, the simulations could be subject to noise accumulation. To fight this problem, a noise control scheme is applied based on the zonal flow conserving Krook operator described in [12]. The value of the Krook coefficient adopted here is $1 / 20$ the linear growth rate of the instabilities, ensuring that the physically relevant features of the system are only weakly affected. Moreover, the signal-to-noise ratio Fig. 4 (lower plot) is used to keep track of the reliability of a simulation. When this value
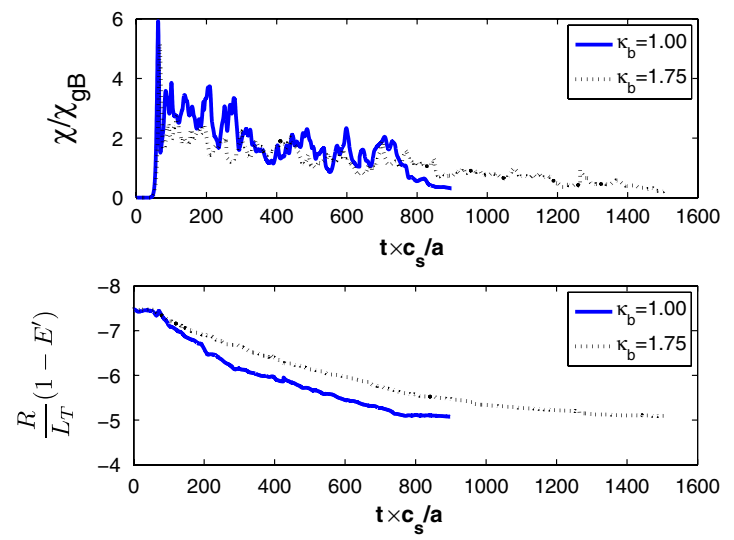

FIG. 3 (color online). Global simulations of ion diffusivity (top) and temperature gradient length (bottom) evolution as a function of time. Both quantities are averaged over the radial interval $0.3<r<0.7$.

approaches $10 \mathrm{~dB}$, the simulation is stopped and a new one is started with an initial temperature gradient value close to the final one of the previous simulation. Figure 4 (upper plot) shows that the ion thermal diffusivity $\chi$ of the new simulation is superposed to the old one after a saturation overshoot. This is a consequence of the turbulent saturated regime not depending on the initial conditions. Figure 4 demonstrates that this feature is correctly captured by the ORB5 code allowing a chain of simulations to follow the evolution of $\chi$ until the relaxation of the temperature gradient brings the system close to the marginal stability. In Fig. 3 the chain of simulations is concatenated, with the overshoot phases omitted. The $\chi$ evolution in the early linear phase of ITG turbulence development shows that the linear drives in the two elongation cases are indeed equal when the same effective temperature gradient scale length is set. But when at a later time the turbulence reaches the saturated state, different values of the transport coefficient
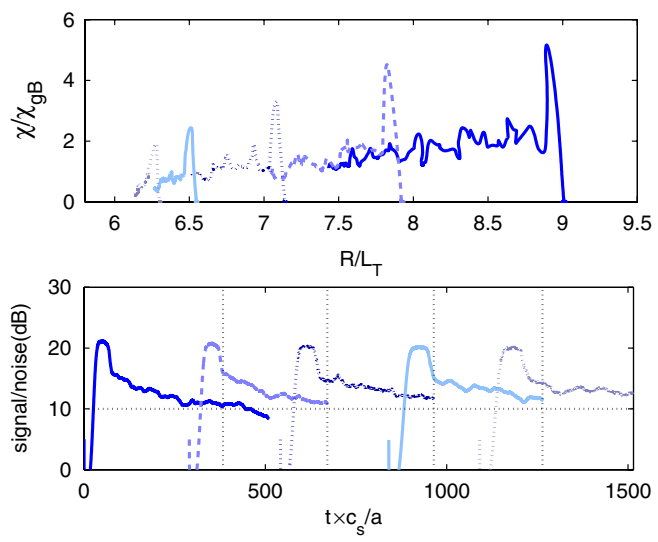

FIG. 4 (color online). Chain of global nonlinear simulations for the case with elongation $\kappa=1.75$. The ion diffusivity is plotted as a function of the gradient length (top) and the signalto-noise ratio is plotted as a function of time (bottom). The times at which the simulations are concatenated are marked by vertical dotted lines on the signal-to-noise plot. 


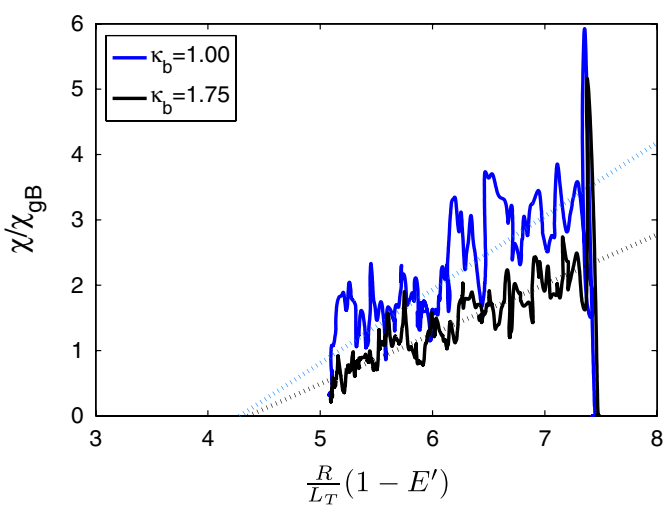

FIG. 5 (color online). Normalized ion diffusivity $\chi / \chi_{\mathrm{gB}}$ plotted as a function of the effective temperature scale length $R / L_{T}\left(1-E^{\prime}\right)$ for $\kappa_{b}=1$ and 1.75 . The dotted lines represent a linear fit of the numerical results omitting the initial overshoot phases.

are found. The relaxation is faster for the low elongation cases, due to the larger thermal diffusivity. The result is that, after a certain time, the diffusion coefficients became similar, since the ITG turbulence comes closer to marginal stability. It is to be noted, however, that at this later time, similar values of transport are found for quite different values of the temperature gradient. The gradient is larger for higher elongation, thus indicating an improvement in confinement. A simple transport model [13] can help us to get more insight. Under the hypothesis of a turbulent transport characterized by an electrostatic gyro-Bohm scaling law, the ion thermal diffusivity can be written under the form:

$$
\frac{\chi_{T}}{\chi_{\mathrm{gB}}}=\chi_{s} q_{s}^{v} \frac{a}{R}\left(\frac{R}{L_{T}}-k_{c}\right) \mathcal{H}\left(\frac{R}{L_{T}}-k_{c}\right) .
$$

The gyro-Bohm diffusivity is used as normalization value $\chi_{\mathrm{gB}}=\rho_{s}^{2} c_{s} / a$, where $\rho_{s}$ is the sound Larmor radius and $c_{s}$ is the sound speed. In Eq. (2), $\nu$ is a coefficient derived from the experimental data and $\mathcal{H}(x)$ is a Heaviside function. This model expresses the ion diffusivity as a function of the distance of the temperature gradient length from a critical value $k_{c}$. The other parameter, $\chi_{s}$, is related to the temperature profile stiffness, or in other words, the resilience of the system to increase the gradient when a larger heat source is applied. It has been shown that, in the presence of large scale flows, the critical gradient is upshifted as compared to the linear threshold, a nonlinear effect called Dimits shift [14]. Keeping in mind the results from linear simulations presented in the previous paragraph, the model represented by Eq. (2) can be adapted to include the linear effects of elongation, when we replace the temperature gradient length with its effective value: $R / L_{T} \rightarrow R / L_{T}\left(1-E^{\prime}\right)$. Nonlinear effects can be kept into account by increasing the stiffness $\chi_{s}$ or by an additional Dimits shift. In Fig. 5 the dotted lines represent a linear fit of the turbulent $\chi$ as a function of the effective temperature gradient. A comparison of these fits with the model Eq. (2) shows that the $\chi_{s}$ value decreases with increasing elonga- tion, while the critical gradient does not show a significant dependence on $\kappa$. A similar study with $\kappa=1.0,1.25,1.5$, 1.75 has been performed for a smaller system size $(\rho=$ $1 / 184$ ) and yields the same conclusions; i.e., the nonlinearly up-shifted gradient is unchanged whereas the stiffness is reduced, when expressed in terms of an effective gradient. In Ref. [6], a Dimits shift depending on the plasma geometry has been reported. But, in the nonlinear simulations presented in [6], the triangularity has been varied along with the elongation; this fact makes it difficult to extrapolate the role of the elongation alone and to compare with our results. However, in both cases an intrinsically nonlinear reduction of ITG transport with elongation is observed, which can be interpreted as follows. It has been shown in [8] that increasing elongation increases both the undamped ZF residual and the geodesic acoustic mode (GAM) damping. Because the quasisteady $\mathrm{ZF}$ is more effective than the oscillatory GAM in suppressing ITG modes by its shearing effect [15], the resulting heat transport is decreased. It should be noted that increasing the plasma current at a fixed elongation was shown to have a similar effect in [16].

To summarize, the favorable effect of plasma elongation on confinement has been obtained from first principles based global linear and nonlinear gyrokinetic direct numerical simulations of ITG turbulence. This effect is attributable to both linear and nonlinear mechanisms. First, the linear drive is reduced; i.e., the same linear drive is obtained for an increased gradient scale $R / L_{T}\left(1-E^{\prime}\right)$ as shown in Fig. 2. Second, nonlinear saturation mechanisms are modified so as to reduce the stiffness of the transport while the nonlinear critical gradient is unchanged, as shown in Fig. 5, when expressed in terms of the increased gradient scale. Finally, it must be stressed that these results have been obtained in a series of noise-controlled simulations.

[1] Y. Miura et al., Nucl. Fusion 32, 1473 (1992).

[2] G. Rewoldt et al., Phys. Fluids 25, 480 (1982).

[3] D. D. Hua et al., Phys. Fluids B 4, 3216 (1992).

[4] R. E. Waltz et al., Phys. Plasmas 6, 4265 (1999).

[5] J. E. Kinsey et al., Phys. Plasmas 14, 102306 (2007).

[6] E. A. Belli et al., Phys. Plasmas 15, 092303 (2008).

[7] A. Kendl and B. Scott, Phys. Plasmas 13, 012504 (2006).

[8] P. Angelino et al., Phys. Plasmas 15, 062306 (2008).

[9] S. Jolliet et al., Comput. Phys. Commun. 177, 409 (2007).

[10] H. Lütjens et al., Comput. Phys. Commun. 97, 219 (1996).

[11] J. W. Connor and L. Chen, Phys. Fluids 28, 2201 (1985).

[12] B. F. McMillan et al., Phys. Plasmas 15, 052308 (2008).

[13] X. Garbet et al., Plasma Phys. Controlled Fusion 46, 1351 (2004).

[14] A. M. Dimits et al., Phys. Plasmas 7, 969 (2000).

[15] T. S. Hahm et al., Phys. Plasmas 6, 922 (1999).

[16] P. Angelino et al., Plasma Phys. Controlled Fusion 48, 557 (2006). 